\title{
Lacking Evidence to Recommend Neoadjuvant Chemotherapy and Definitive Radiotherapy in Muscle-Invasive Bladder Cancer
}

\author{
In response to: Trimodality Therapy for Muscle-Invasive Bladder Cancer: Recent Advances and \\ Unanswered Questions published by Di Maria Jiang et al. Current Oncology Reports 2020, \\ https://doi.org/10.1007/s11912-020-0880-5
}

\author{
Dirk Böhmer $^{1}$ (D) - Arne Grün ${ }^{2}$ (D) \\ Accepted: 18 December 2020 / Published online: 20 January 2021 \\ (C) The Author(s) 2021
}

\begin{abstract}
We comment on the paper of Di Maria Jiang et al. published in Current Oncology Reports 2020, https://doi.org/10.1007/s11912020-0880-5. We disagree on a major recommendation of the authors because of lacking evidence. This response is considered to be important for readers of Current Oncology Reports.
\end{abstract}

Keywords Bladder cancer · Trimodality treatment · Neoadjuvant chemotherapy

Letter to the editor:

We read with great interest the article by Jiang et al. on their review regarding trimodality therapy in advanced muscleinvasive bladder cancer (MIBC). They comprehensively address issues on bladder preservation strategies from the past to present. They present an elaborate view into the future with modern immunotherapy concepts and possible genetic pathway targets. The authors state that neoadjuvant chemotherapy (NAC) is the preferred setting for all patients regardless of whether patients receive radiotherapy or cystectomy.

We strongly disagree with the authors' interpretation of results regarding neoadjuvant chemotherapy and subsequent chemoradiation for the following reasons.

Only one randomized trial is available with NAC involving patients who are eligible for cystectomy or chemoradiation. Patients in the BA06 30894 trial were randomized to either neoadjuvant chemotherapy or no chemotherapy. The type of

Dirk Böhmer

dirk.boehmer@charite.de

1 Department of Radiation Oncology, Charité University Medicine, Campus Benjamin Franklin, Hindenburgdamm 30,

12203 Berlin, Germany

2 Department of Radiation Oncology, Charité University Medicine, Campus Virchow Klinikum, Berlin, Germany definitive treatment was not randomized but the choice of the patient and/or physician. Although the numbers of randomized patients receiving radiotherapy (RT) $(N=403)$ and cystectomy $(N=428)$ seem almost evenly distributed the BA06-authors clearly point out that several factors, namely WHO performance status, age, number of $\mathrm{T} 2$ tumors, and number of N0 patients, respectively, were unbalanced among both groups.

Results show a significant reduction in the risk of death for the cystectomy group (HR: $0.74 ; p=0.022$ ). Risk reduction for the radiotherapy group is in fact $20 \%$ (HR: 0.80 ) but this difference is not statistically significant $(p=0.070)$.

Locoregional disease-free survival is improved again by $26 \%$ for the cystectomy group $(p=0.019)$ but only by $9 \%$ for the RT group with $p=0.417$ [1].

The second phase III trial, RTOG 89-03, evaluated neoadjuvant chemotherapy before trimodality treatment. Due to a high rate of chemotherapy related toxicities, it closed prematurely. With 123 patients randomized neither overall survival nor metastases-free survival were significantly different [2]. The remaining studies presented by the authors are either phase II trials, cohort-studies, or ongoing studies without published results. Furthermore, the authors do not add specific evidence added to support their assumption.

Thus, it is our belief that the conclusion to prefer neoadjuvant chemotherapy in patients who undergo bladder-sparing approaches combined with radiotherapy is not supported by any high-level evidence. 
The authors should acknowledge that neoadjuvant chemotherapy is not considered standard in patients undergoing trimodality treatment for muscle-invasive bladder cancer.

Authors' Contributions D. B. and A. G. are equally responsible for the content of this letter.

Funding Open Access funding enabled and organized by Projekt DEAL.

\section{Compliance with Ethical Standards}

Conflict of Interest The authors declare that they have no conflict of interest.

Ethics Approval Not applicable.

Consent to Participate Not applicable.

Consent for Publication Both authors consent to publication of their work.

Data Availability Not applicable.

Code Availability Not applicable.

Open Access This article is licensed under a Creative Commons Attribution 4.0 International License, which permits use, sharing, adaptation, distribution and reproduction in any medium or format, as long as you give appropriate credit to the original author(s) and the source, provide a link to the Creative Commons licence, and indicate if changes were made. The images or other third party material in this article are included in the article's Creative Commons licence, unless indicated otherwise in a credit line to the material. If material is not included in the article's Creative Commons licence and your intended use is not permitted by statutory regulation or exceeds the permitted use, you will need to obtain permission directly from the copyright holder. To view a copy of this licence, visit http://creativecommons.org/licenses/by/4.0/.

\section{References}

1. International Collaboration of Trialists on behalf of the Medical Research Council Advanced Bladder Cancer Working Party (now the National Cancer Research Institute Bladder Cancer Clinical Studies Group), the European Organisation for Research and Treatment of Cancer Genito-Urinary Tract Cancer Group, the Australian Bladder Cancer Study Group, the National Cancer Institute of Canada Clinical Trials Group, Finnbladder, Norwegian Bladder Cancer Study Group, and Club Urologico Espanol de Tratamiento Oncologico Group. International Phase III Trial Assessing Neoadjuvant Cisplatin, Methotrexate, and Vinblastine Chemotherapy for Muscle-Invasive Bladder Cancer: Long-Term Results of the BA06 30894 Trial. J Clin Oncol. 2011;29:2171-7.

2. Shipley WU, Winter KA, Kaufman DS, et al. Phase III trial of neoadjuvant chemotherapy in patients with invasive bladder cancer treated with selective bladder preservation by combined radiation therapy and chemotherapy: initial results of radiation therapy oncology group 89-03. J Clin Oncol. 1998;16(11):3576-83.

Publisher's Note Springer Nature remains neutral with regard to jurisdictional claims in published maps and institutional affiliations. 